\title{
Lactobacillus-containing probiotic supplementation increases Helicobacter pylori eradication rate: Evidence from a meta-analysis
}

\author{
Xi Zheng, Lin Lyu and Zhechuan Mei
}

Department of Gastroenterology. Second Affiliated Hospital of Chongqing Medical University. Chongqing Medical University. Chongqing, China

\begin{abstract}
Background: Helicobacter pylori infection is recognized as a major contributory factor to many diseases, but recommended eradication therapies demonstrated unsatisfactory eradication rates. Currently, some studies suggested that lactobacillus species have an inhibitory action on Helicobacter pylori both in vitro and in vivo.
\end{abstract}

Objective: this meta-analysis broadly examined the efficacy of eradication regimens supplemented with lactobacillus-containing probiotic on eradication rates and side effects.

Methods: eligible articles were identified by comprehensive searches. Statistical analysis was performed with Review Manager 5.2. Outcomes were finally evaluated according to GRADE system.

Results: nine randomized controlled trials of high-quality met eligible criteria. Risk ratio of eradication was available for 1,163 patients. Lactobacillus-containing probiotics significantly increased the eradication rate compared with the control group based upon intention-to-treat analysis [RR $=1.14 ; 95 \% \mathrm{CI}(1.06-1.22)$; number needed to treat $(\mathrm{NNT})=10$ ] by the fixed effect model without significant publication bias, but no significant reduction associated with overall side effects was observed [RR $=0.88$; $95 \% \mathrm{CI}(0.73$ 1.06)]. In the subgroup analysis, eradication rates raised significantly by $17 \%$ in lactobacillus administrated alone group [RR $=1.25 ; 95 \% \mathrm{CI}(1.13-1.37) ; \mathrm{NNT}=6]$. In multistrain probiotics group, eradication rates enhanced only $2.8 \%[\mathrm{RR}=1.04 ; 95 \% \mathrm{CI}$ (0.94-1.14)]. It also showed that lactobacillus-containing probiotics improved the eradication rates, respectively, both in adults $[\mathrm{RR}=$ 1.12 ; $95 \% \mathrm{CI}(1.04-1.20)$; NNT $=12]$ and in children $[\mathrm{RR}=1.25$; $95 \% \mathrm{CI}(1.01-1.53) ; \mathrm{NNT}=7]$.

Conclusions: Lactobacillus-containing probiotic as an adjunct is effective to eradication therapy, while side effects caused by eradication treatment may not decrease. Furthermore, lactobacillus administrated alone will distinctly benefit eradication therapy.

Key words: Helicobacter pylori. Meta-analysis. Probiotics.

Accepted: 12-09-2013

Correspondence: Zhechuan Mei. Department of Gastroenterology. Second Affiliated Hospital of Chongqing Medical University. Chongqing Medical University. Chongqing, China e-mail:675614129@ qq.com

Zheng X, Lyu L, Mei Z. Lactobacillus-containing probiotic supplementation increases Helicobacter pylori eradication rate: Evidence from a meta-analysis. Rev Esp Enferm Dig 2013;105:445-453. 
rence of $H$. pylori may be inhibited.

Currently, lactobacillus has been demonstrated anti- $H$. pylori activity in vitro and in animal models of $H$. pylo$r i$ infection (8-11). Several clinical trials have reported the great therapeutic value of lactobacillus preparations in anti- $H$. pylori regimen. Deguchi et al. (12) indicated a significant improvement in eradication rate with yogurtplus-triple treatment (PPI + amoxicillin + clarithromycin + probiotic for 7 days). Ojetti et al. (13) also concluded the result that the eradication rate of second-line triple therapy raised by $18 \%$ with probiotic supplementation, and the incidence of nausea and diarrhea reduced distinctly. However, the addition of probiotics obtained a significant decrease of side effects, while there was no statistical alteration of eradication in the trial of Manfredi (14). The study of Medeiros et al. (15), administrating L. acidophilus as a supplement to triple therapy also showed no benefit on eradication. Yoon et al. (16) conducted a triple therapy with probiotic for 14 days (probiotic + PPI + moxifloxacin + amoxicillin), which neither enhanced eradication rate nor lowered the overall adverse effects. The previous meta-analyses such as Zou et al. (17) demonstrated that Lactobacilli-containing probiotic as an adjunctive agent conferred a positive impact on improving eradication rates and reducing therapy-related side effects. The analysis of Wang et al. (18) came to the same conclusion. Nonetheless, there were a few methodological shortcomings in those meta-analyses. So far, many trials have sought to evaluate the efficacy of lactobacillus added in the eradication therapies, but they were dramatically different in quality. At this moment, it is urgent to perform a high quality meta-analysis of randomized controlled trials (RCTs) that compares the eradication rates and side effects of a defined probiotic preparation with those of placebo (or blank control) in $H$. pylori-positive participants.

\section{METHODS}

\section{Selection of studies}

Trials were identified by systematically searching 6 electronic databases (Pubmed, Embase, Web of Science, The Cochrane Central Register of Controlled Trials, Google Scholar and Chinese Biomedical Database), by time up to October 2012. The following strategy was used to find eligible trials: "randomized controlled trials" or "controlled clinical trials" or "clinical trials" or "random allocation" or "double-blind method" or "single-blind method" or "placebo") and [("Helicobacter pylori" or "H. pylori") and ("lactobacillus" or "probiotic" or "probiotics" or "yeast" or "yogurt")]. Besides, the reference lists from retrieved articles and abstracts of conference proceedings were also searched to identify the relevant studies. Other studies were attempted to be screened by contacting known authors of some identified trials. The unpublished stud- ies were retrieved from the Clinical Trials. gov registry. Two reviewers (Z.X \& L.L) independently screened the database search for titles and abstracts. If either reviewer determined the title and abstract meeting eligible criteria, the full text of the study was retrieved.

\section{Criteria for considering studies}

\section{Inclusion criteria}

- Only RCTs of high quality were included, with clear and adequate methods of randomization. Loss-up rates were less than $20 \%$.

- Participants were assessed by ${ }^{13} \mathrm{C}$-urea breath test, histology, and stool antigen test. At least one positive test result was considered as confirmation of infection.

- Probiotics preparations and proton pump inhibitor plus two antibiotics were used in the intervention group in triple therapy. On each stage of sequential treatment, two antibiotics were allowed at most. The control group received the same eradication regimen with or without placebo.

- The primary outcome was the rate of $H$. pylori eradication, which had to be confirmed at least 4 weeks after treatment. The secondary outcome was the frequency of overall adverse effects.

\section{Exclusion criteria}

- RCTs of high risk of bias were excluded, as well as the methods of randomization being unclear or inadequate. Quasi-randomized controlled trials were also excluded.

- Participants had a history of drugs use for $\mathrm{H}_{2}$ receptor antagonists or proton pump inhibitors, with concurrent cardiovascular diseases, immunologic diseases or upper respiratory tract infections in the past 3 months.

- Probiotics contain Saccharomyces boulardii, Bacillus subtilis, Bacillus lausii or Clostridium butyrium.

- Subsets of cases from a previously published article by the same author were excluded.

\section{Quality assessment}

Evaluation tools used to be selected such as Jadad scale, were not chosen due to the problems and defects with use (19), and as strongly recommended in the Cochrane Handbook 5.1, the Cochrane collaborations' tool for inspecting the risk of bias was performed (20). The study was considered to be low risk of bias if each of the bias items was low risk. On the contrary, study was judged as high risk of bias in the situation that one of the items was high risk. Two investigators independently gave each eligible study an overall rating of high, low, or unclear risk of bias, if dis- 
agreements emerged, and then consulted a third reviewer to obtain the final option.

\section{Data extraction}

Two reviewers (Z.X. \& L.L.) independently abstracted relevant information from each eligible study depending on a standardized data abstraction sheet. Data were extracted pertaining to the location of trials, character of enrolled participants, initial/rechecking methods for assessing $H$. pylori infection, strain and course of probiotic treatment, $H$. pylori eradication regimens, follow-up time, and loss-up rate. Synchronously, the key outcomes recorded including eradication rates and incidence of total side effects were abstracted from all included studies. Disagreements between reviewers regarding data collection were resolved through discussion. The authors of the studies were contacted by e-mail and telephone for additional information if necessary.

\section{Statistical analysis}

Data were discreetly entered into RevMan 5.2. The dichotomous outcomes were statistically analyzed by Risk ratio (RR), and the results were reported with $95 \%$ confidence intervals (CIs). Eradication rates and side effects were analyzed based on a fixed-effects model using the method of Mantel-Haenszel by Intention-to-treat (ITT) analysis. Number needed to treat (NNT) were calculated if there was significant statistical difference in the outcome between experiment group and control group.

Heterogeneity between the studies was assessed by chisquare test and $\mathrm{I}^{2}$ statistic. If $\mathrm{p}<0.1$ and $/$ or $\mathrm{I}^{2}>50 \%$, there was substantial heterogeneity. Moreover, $\mathrm{I}^{2}$ was used to evaluate the level of heterogeneity $(0-30 \%$ : homogeneity; 30-50 \%: moderate heterogeneity; 50-80 \%: substantial heterogeneity; > $80 \%$ : considerable heterogeneity). The sensitivity analysis was further examined by evaluating whether the exclusion study impacted on the pooled results.

For the primary outcome, planned subgroup analyses were performed based on species of probiotics, age and eradication regimens. Publication bias was assessed by a funnel plot (20). Once outcomes were all evaluated, a summary of findings table was created according to the GRADE system $(21,22)$.

\section{RESULTS}

\section{Result of the search}

The selection of eligible studies is outlined in figure 1 . Using bibliographical search strategy and yielding a total of 295 citations, of which 195 were not RCTs and 2 were duplicate reports of the same study. We excluded another

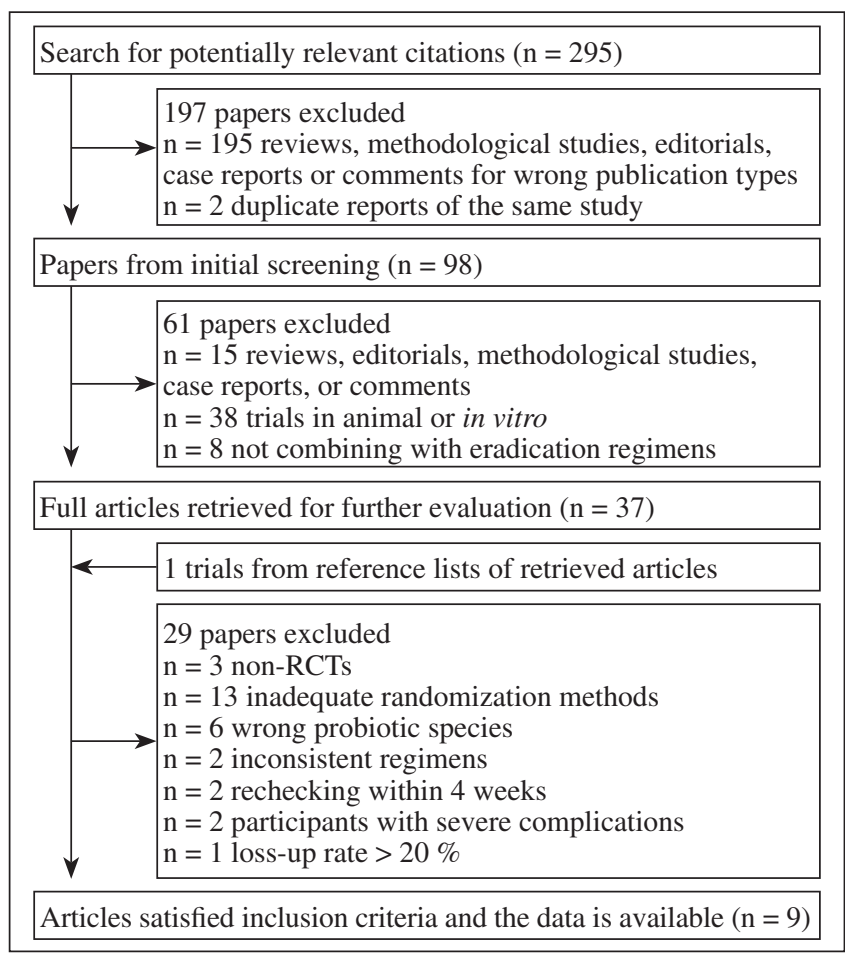

Fig. 1. Flowchart for selection of studies.

61 out from 98 articles through further screening (15 studies being non-corresponding publication type, 38 trials in animal or in vitro, 8 studies not combining with eradication regimens). At this point, full texts of the remaining 37 studies were retrieved for review. The reference lists from retrieved articles were searched. As a result, one relevant study could not be found in the database, but finally the full article was obtained by contacting the author.

Furthermore, one author of the eligible studies informed us of their plan for further relevant study on the step of making a placebo. Of the 38 full text articles (37 articles plus 1 trial mentioned above), 29 studies were excluded ( 3 trials being non-RCTs, 13 trials using inadequate randomization methods, 6 trials with wrong intervention, 2 trials with non-corresponding control group, 2 studies rechecking $H$. pylori infection within 4 weeks after treatment, patients in 2 trials with severe complications, and 1 trial having the loss-up rate more than $20 \%$ ).

\section{Characteristics of included studies}

Then identified studies were published between 2000 and 2012, all of which were conducted in Europe, Asia, and South America. There was 1 multicenter trial included (23). The sample size for the eligible studies ranged from 40 to 337 patients and a total of 1,163 patients were identified including adults and children. The characteristics of 9 trials selected in the meta-analysis are summarized in detail in table I. 
Table I. Baseline characteristics of included studies

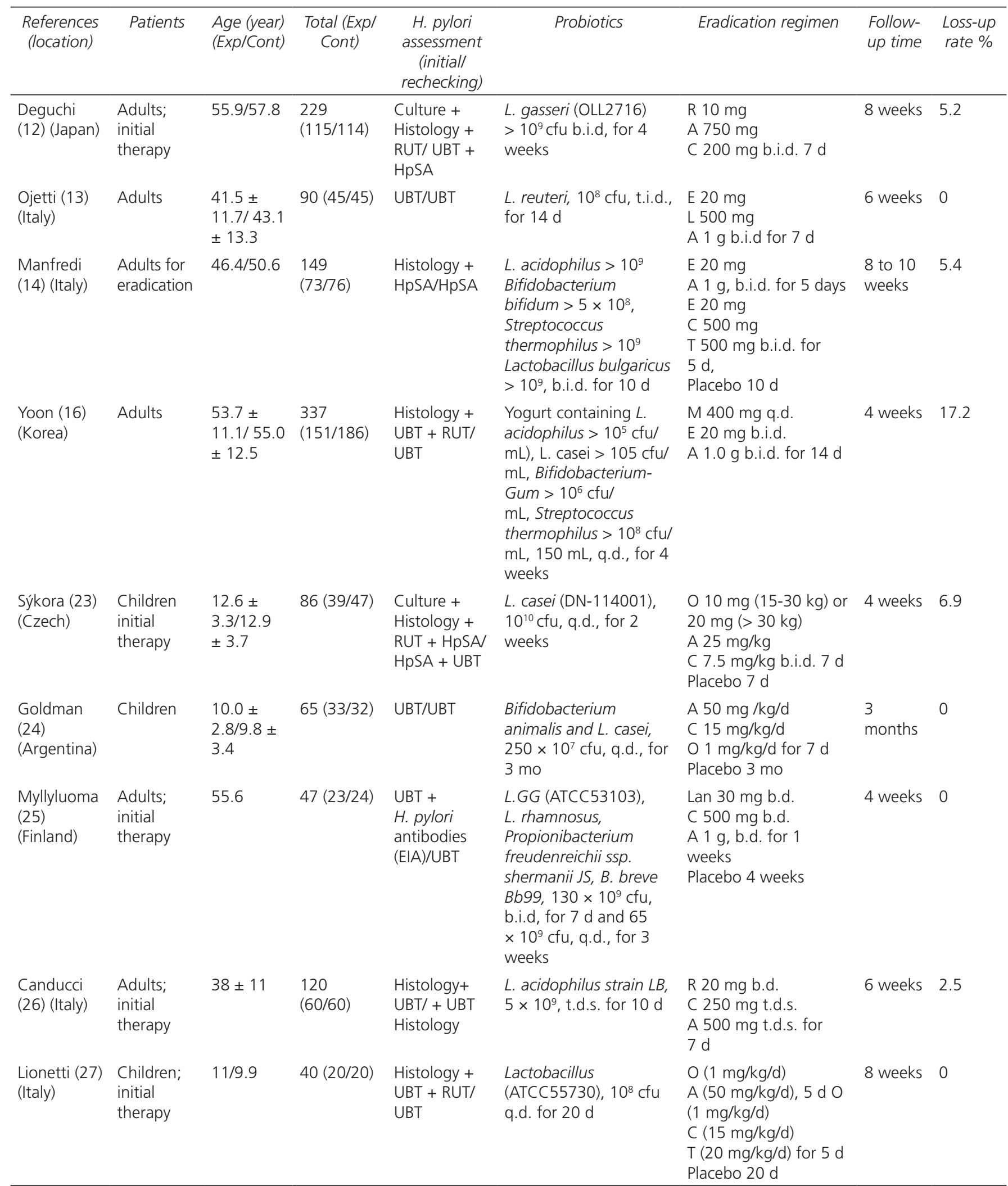

A: Amoxicillin; E: Esomeprazol; C: Clarithromycin; T: Tinidazole; R: Rabeprazole; O: Omeprazole; L: Levofloxacin; Lan: Lansoprazole; M: Moxifloxacin; RUT: Rapid urease test. HpSA: H. pylori stool antigen test; UBT: ${ }^{13} \mathrm{C}$-urea breath test; Exp: Experiment group; Cont: Control group. 


\section{Risk of bias assessment of included studies}

All included studies performed a conventional parallel group design. Of all trials, sequence generation (method of randomization) was clear and appropriate. Concealment was unclear in 7 studies $(12,13,16,23-26)$, which was performed by using appropriately sealed boxes in 2 studies $(14,27)$. Knowledge of the assigned intervention may impact on behavioral outcomes (such as number of clinic visits) but not on physiological outcomes. Thus, assessments of risk of bias resulting from lack of blinding may need to be made separately for different outcomes (20). Blinding of participants and outcome assessment were regarded as low risk by system evaluation personnel because the first outcome in this study was physiological (Figs. 2 and 3).

\section{Eradication rates}

Risk ratios for eradication rates were available for all selected trials. Of these included studies, 4 studies reported significantly enhancing eradication rates, and the remaining 5 studies had similar effect. The pooled RR in the experiment versus control group was 1.14 [95 \% CI (1.06-1.22); $\mathrm{p}=0.0002]$ by choosing the fixed effects model (Fig. 4). The fixed effects model was used for the reason that there



Fig. 2. Risk of bias summary: Each risk of bias item for each included study. "+" indicates a low risk of bias, "?" indicates unclear a risk of bias. was no significant heterogeneity emerged among the studies [Cochran's $\mathrm{Q}=11.67 ; \mathrm{p}=0.17 ; \mathrm{I}^{2}=31 \%$ ]. The corresponding NNT was 10 . To evaluate the stability of the results of this meta-analysis, we conducted a sensitivity analysis by estimating the pooled RRs in the absence of each study included in turn. It turned out that there was no significant change observed on account of exclusion of any studies.

\section{Side effects}

Total side effects were also conducted for meta-analysis. There were 5 cases (346 patients in the experiment group and 393 patients in the control group) displaying the data for total side effects rates. Using the fixed effects model, the pooled RR by ITT analysis for the probiotic group versus control group was not significant $[\mathrm{RR}=0.88 ; 95 \%$ CI (0.73-1.06)], while the heterogeneity was acceptable [Cochran's $\mathrm{Q}=7.82 ; \mathrm{p}=0.10 ; \mathrm{I} 2=49 \%$ ] (Fig. 5).

\section{Subgroup analysis}

Since subgroup analysis would be beneficial for clinical conclusions, the primary outcome of eradication in the identified studies was stratified based on species of probi-

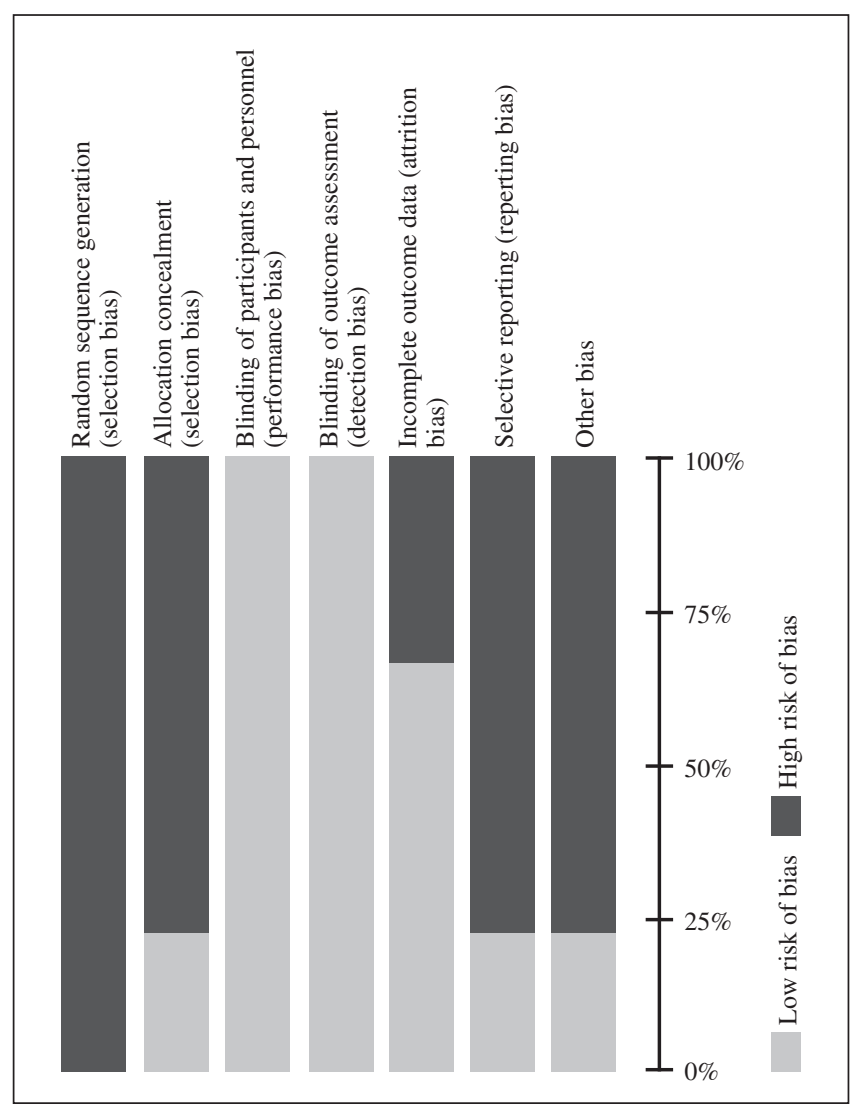

Fig. 3. Risk of bias graph: review authors' judgements about each risk of bias item presented as percentages across all included studies. 


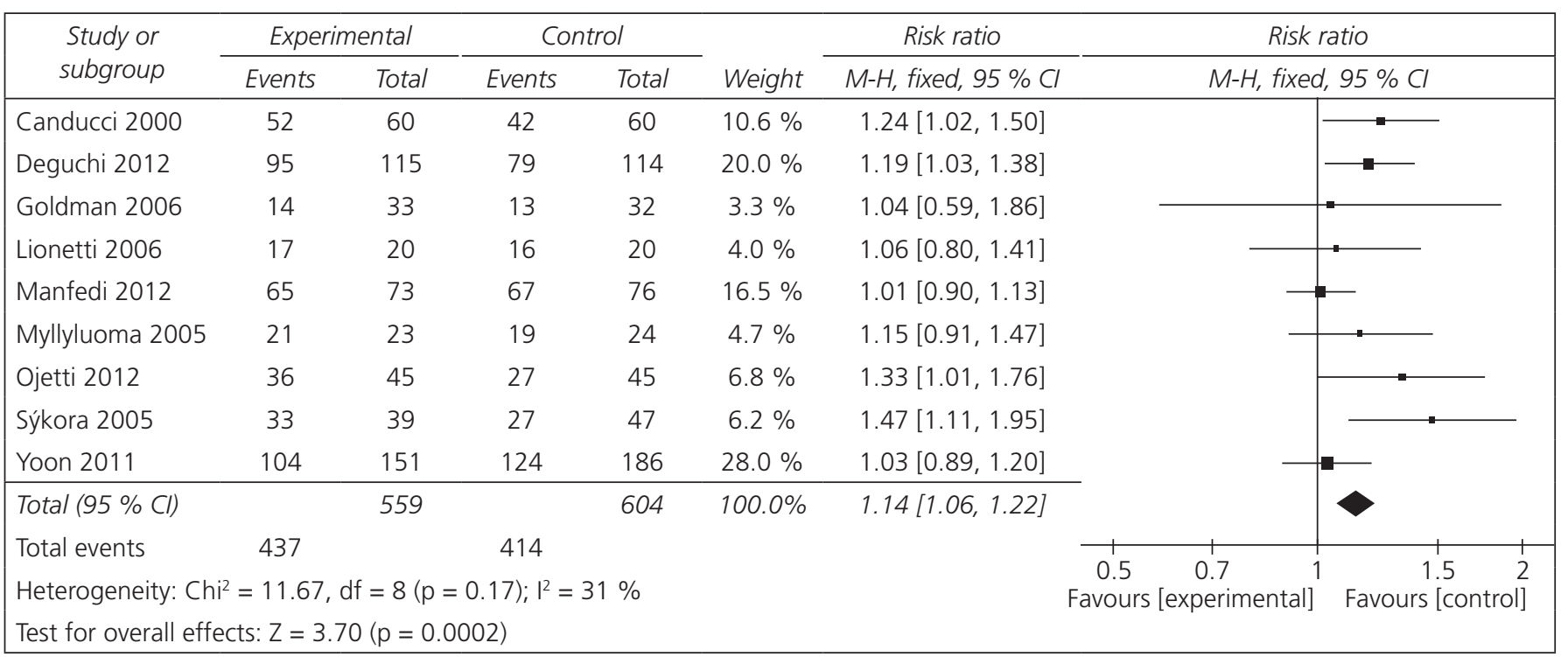

Fig. 4. Eradication rate with or without probiotic.

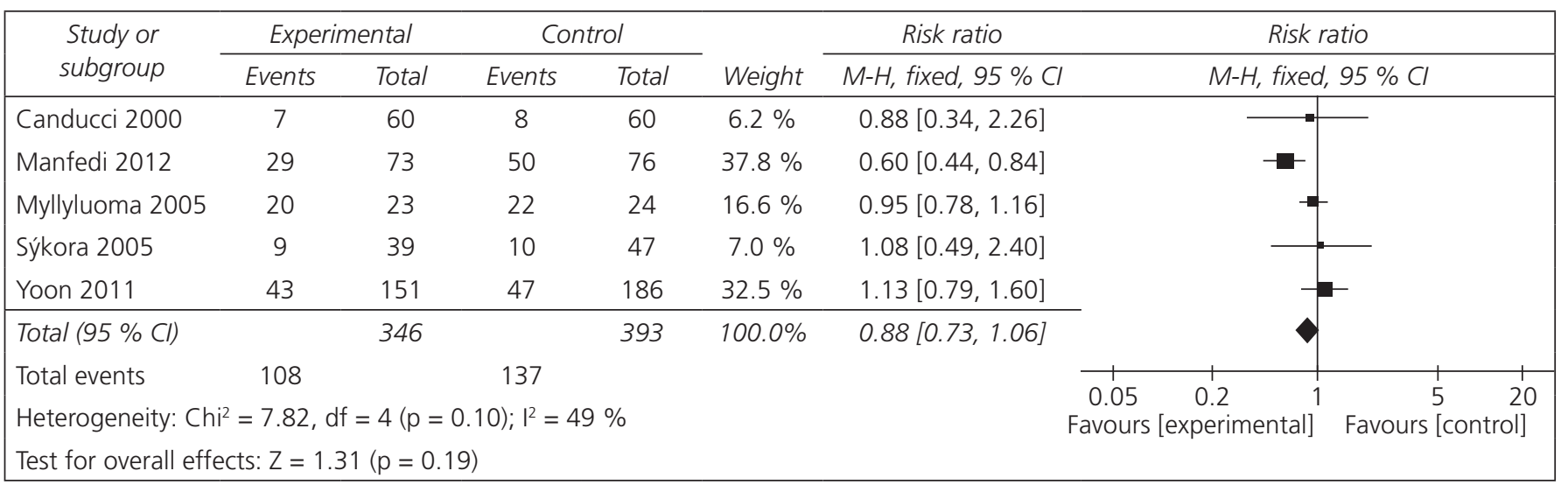

Fig. 5. Overall side effect with or without probiotic.

otics (lactobacillus used alone vs. multistrain probiotics), age (adults $v s$. children) and eradication regimens (triple regimen $v s$. sequential regimen). Probiotic preparations improved eradication rates in each group except in patients prescribed multistrain probiotics [72.9 vs. $70.1 \%$; RR = 1.04; $95 \% \mathrm{CI}(0.94-1.14)]$ and sequential regimen [88.2 vs. $83.8 \% ; \mathrm{RR}=1.02 ; 95 \% \mathrm{CI}(0.92-1.14)]$.

\section{Assessment of publication bias}

A funnel plot of studies for the first outcome was made to detect publication bias (20). It suggested a symmetric distribution around the effect estimate, indicating minimal publication bias in the literature for studies evaluating efficacy of probiotics in eradication therapy. However, less than 10 studies were included, in this condition, the funnel plot may not truly reflect publication bias (28).

\section{DISCUSSION}

\section{Summary of evidence}

The results revealed that lactobacillus-containing probiotic supplementation potentially elevated $H$. pylori eradication rates by approximately $10 \%$, but side effects along with eradication treatment might not reduce significantly. Furthermore, in the subgroup analysis, eradication rates increased significantly by almost $17 \%$ in lactobacillus used alone group. The eradication rates of patients administrated for lactobacillus-containing probiotics plus triple regimens significantly achieved an increase of $11 \%$ compared with the control group, while sequential regimens supplemented with probiotics might not show significant improvement. In this meta-analysis, administrating lactobacillus-containing probiotics with the conventional regimens seemed to be effective in the eradication therapies both in children and adults. 
In the end, using the outcomes reviewed, a summary of findings table has been made on the basis of GRADE system $(21,22)$ (Table II). For the primary outcome, that probiotics combined with traditional eradication increased the eradication rates was graded as moderate quality, and it was evaluated as strong recommendation. As the second endpoint, probiotics reducing the chances of side effects was graded as low quality.

\section{Limitations of the study}

In order to perform a meta-analysis of high quality, the studies seemed methodologically reasonable with regard to randomization, loss-up rate $(<20 \%)$ and ITT analysis. Overall, no study of high risk of bias was included in our study. Potential limitations included unclear risk of bias of allocation concealment, attention and reporting, because of lack of adequate information to assess the methodology of included studies. Some trials of no blinding were included, as assessments of blinding bias resulting from lack of blinding may need to be made separately for different outcomes, the interventions may not impact on physiological outcomes (20). In general, all bias referred above could not obviously have an influence on the outcomes. Bibliography retrieval was comprehensive for all included trials indexed in databases. However, there was a lack of research reported in North America, which was apparently another limitation. No obvious publication bias was detected in this study. Although probiotics may increase the eradication rate, they perhaps exert intricate influence on patients due to the complicated mechanism. Besides, considering the cost added to eradication therapy, the utilization of probiotics in clinical practice are probably limited.

\section{Agreement and disagreement with other studies}

It was confirmed in our study that lactobacillus-containing probiotic remarkably improved $H$. pylori eradication rates but the occurrence of total side effects did not significantly decrease. The former outcome agreed with other meta-analyses $(17,18,29)$, but the latter was inconsistent with the studies which suggested significant reduce of side effects. The following was a summary of the rational points. First, all the previous reviewers pooled odds ratios for the analysis, while we used risk ratios for data pooling. In the case that events are common, as is in the relevant clinical trials, the differences between odds and risks are large. When interventions increase the incidence of events, the odds ratio will be larger than the risk ratio. Likewise, for interventions that reduce the incidence of events, the odds ratio will be smaller than the risk ratio, so the misinterpretation will tend to overestimate the intervention effect, especially in the condition that events are common (for example, risks of events more than $20 \%$ ). This error in interpretation is pointed out in Cochrane handbook, which is quite common in published reports (20). Second, pooling data from different genera of probiotics which are presumed with variations in their native intestinal microbial activity, may result in erroneous conclusions, and the

Table II. GRADE assessment for eradication regimens with or without probiotic for Helicobacter pylori eradication

\begin{tabular}{|c|c|c|c|c|c|c|}
\hline \multirow[t]{3}{*}{ Outcomes } & \multicolumn{2}{|c|}{$\begin{array}{l}\text { Illustrative comparative risks* } \\
\qquad(95 \% \mathrm{Cl})\end{array}$} & \multirow[t]{3}{*}{$\begin{array}{l}\text { Relative effect } \\
\qquad(95 \% \mathrm{Cl})\end{array}$} & \multirow[t]{3}{*}{$\begin{array}{l}\text { No. of participants } \\
\text { (studies) }\end{array}$} & \multirow[t]{3}{*}{$\begin{array}{l}\text { Quality of the } \\
\text { evidence (GRADE) }\end{array}$} & \multirow[t]{3}{*}{ Importance } \\
\hline & Assumed risk & $\begin{array}{l}\text { Corresponding } \\
\text { risk }\end{array}$ & & & & \\
\hline & Control & $\begin{array}{l}\text { Eradication } \\
\text { regimens with } \\
\text { probiotic }\end{array}$ & & & & \\
\hline \multirow[t]{4}{*}{ Eradication rate } & \multicolumn{2}{|c|}{ Study population } & \multirow{4}{*}{$\begin{array}{l}\text { RR } 1.14 \\
(1.06 \text { to } 1.22)\end{array}$} & \multirow{4}{*}{$\begin{array}{l}1,163 \\
\text { (9 studies) }\end{array}$} & \multirow{4}{*}{$\begin{array}{l}\oplus \oplus \oplus \Theta \\
\text { moderate }\end{array}$} & \multirow[t]{4}{*}{ Critical } \\
\hline & 685 per 1000 & $\begin{array}{l}781 \text { per } 1,000 \\
\text { (727 to } 836)\end{array}$ & & & & \\
\hline & \multicolumn{2}{|l|}{ Moderate } & & & & \\
\hline & 693 per 1000 & $\begin{array}{l}790 \text { per } 1,000 \\
(735 \text { to } 845)\end{array}$ & & & & \\
\hline \multirow[t]{4}{*}{ Side effect } & Study populatic & & \multirow{4}{*}{$\begin{array}{l}\text { RR } 0.88 \\
(0.73 \text { to } 1.06)\end{array}$} & \multirow{4}{*}{$\begin{array}{l}739 \\
\text { (5 studies) }\end{array}$} & \multirow{4}{*}{$\begin{array}{l}\oplus \oplus \Theta \Theta \\
\text { low }\end{array}$} & \multirow[t]{4}{*}{ Important } \\
\hline & 349 per 1,000 & $\begin{array}{l}307 \text { per } 1,000 \\
(254 \text { to } 370)\end{array}$ & & & & \\
\hline & \multicolumn{2}{|l|}{ Moderate } & & & & \\
\hline & 253 per 1,000 & $\begin{array}{l}223 \text { per } 1,000 \\
(185 \text { to } 268)\end{array}$ & & & & \\
\hline
\end{tabular}


results could be contrary to other probiotics. In the settings we excluded the probiotics containing Saccharomyces boulardii, Bacillus subtilis, Bacillus clausii or Clostridium butyrium. But this intervention factor was not taken into account by any previous reviewers. Last but not least, significant heterogeneity existed in the pooled data of side effects for the studies of Wang $\left(\mathrm{I}^{2}=84.6 \%, \mathrm{p}=0.000\right)$ and Zou $\left(\mathrm{I}^{2}=62 \%, \mathrm{p}=0.02\right)$. As mentioned above, the discrepancies were reasonably clarified.

\section{Clinical implications}

The results provide important clinical implications and direction for future researches. Therefore, successful eradication of $H$. pylori is a crucial component in the treatment of gastroenterologic and hematologic diseases including chronic gastritis, peptic ulcers, gastric adenocarcinoma, gastric-mucosa-associated lymphoid tissue lymphoma, sideropenic anemia, and primary immune thrombocytopenia. Nevertheless, $H$. pylori eradication rate of first-line triple regimen and rescue regimen with various patterns of antibiotic resistance are from 55 to $90 \%$ and 70 to $90 \%$ throughout the world (30-32). Furthermore, because of the use of antibiotics in eradication therapy, they kill not only H. pylori, but also the normal flora in the stomach, equivalent to further damage of the gastric micro-ecological environment which leads to the bacterial superinfection and $H$. pylori infection recurrence. At this point, probiotics can be helpful to reconstruct the balanced environment.

Therefore several conjectural mechanisms have been proposed to discuss the effects of lactobacillus on $\mathrm{H}$. pylori in vitro and in vivo trials. Firstly, the bacteriocins generated by lactobacillus exert an inhibition on $H$. pylori directly, and some other probiotic bacterium including bifidobacterium and enterococcus may also suppress the viability of $H$. pylori by producing heat stable proteinaceous compounds (33). Secondly, it is concerning the secreted metabolites especially lactic acid produced by lactobacillus suppressing urease activity of $H$. pylori $(34,35)$. Thirdly, the mechanism is that lactobacillus decreased the $H$. pylori load in the gastric mucosa through the stabilization of the mucosal barrier $(36,37)$, to which the mucin secretion is the main approach, and it probably results from mucin gene allele mutation and expression $(35,38,39)$. Another mechanism is proposed to be the immunologic response which reduces inflammatory chemokine expression and lymphocyte infiltration (35,40-42). Iopioid and cannabinoid receptors can also be activated by probiotics, thus the discomfort resulting from antibiotics relieves (43). In the future, researches for finding out the defined species of lactobacillus capable of exerting significant suppression of $H$. pylori, used with the most suitable dose and duration, may greatly benefit eradication treatment.

In conclusion, this is a moderate-quality and strong recommendation evidence, on the basis of GRADE system, that the application of lactobacillus-containing probiotic especially used alone is a safe choice for improving $H$. pylori eradication rates. Thus, lactobacillus used alone should be paid more attention to.

\section{REFERENCES}

1. Sonnenberg A, Lash RH, Genta RM. A national study of Helicobactor pylori infection in gastric biopsy specimens. Gastroenterology 2010;139:1894-901.

2. Lehours P, Zheng Z, Skoglund A, Mégraud F, Engstrand L. Is there a link between the lipopolysaccharide of Helicobacter pylori gastric MALT lymphoma associated strains and lymphoma pathogenesis? PLoS One 2009;4:e7297.

3. Alakkari A, Zullo A, O' Connor HJ. Helicobacter pylori and nonmalignant diseases. Helicobacter 2011;16(Supl.1):33-7.

4. Graham DY, Fischbach L. Helicobacter pylori treatment in the era of increasing antibiotic resistance. Gut 2010;59:1143-53.

5. Wu DC, Hsu PI, Wu JY, Opekun AR, Kuo CH, Wu IC, et al. Sequential and concomitant therapy with four drugs is equally effective for eradication of H. pylori infection. Clin Gastroenterol Hepatol 2010;8:36-41.

6. De Francesco V, Giorgio F, Hassan C, Manes G, Vannella L, Panella C, et al. Worldwide H. pylori antibiotic resistance: a systematic review. J Gastrointestin Liver Dis 2010;19:409-14.

7. García A, Sáez K, Delgado C, González CL. Low co-existence rates of Lactobacillus spp. and Helicobacter pylori detected in gastric biopsies from patients with gastrointestinal symptoms. Rev Esp Enferm Dig 2012;104:473-8.

8. Sunanliganon C, Thong-Ngam D, Tumwasorn S, Klaikeaw N. Lactobacillus plantarum B7 inhibits Helicobacter pylori growth and attenuates gastric inflammation. World J Gastroenterol 2012;18:2472-80.

9. Yang YJ, Chuang CC, Yang HB, Lu CC, Sheu BS. Lactobacillus acidophilus ameliorates $\mathrm{H}$. pylori-induced gastric inflammation by inactivating the smad7 and NFkappaB pathways. BMC Microbiol 2012;12:38.

10. Cui Y, Wang CL, Liu XW, Wang XH, Chen LL, Zhao X, et al. Two stomach-originated lactobacillus strains improve Helicobacter pylori infected murine gastritis. World J Gastroenterol 2010;16:445-52.

11. Isobe H, Nishiyama A, Takano T, Higuchi W, Nakagawa S, Taneike I, et al. Reduction of overall Helicobacter pylori colonization levels in the stomach of Mongolian gerbil by Lactobacillus johnsonii La1 (LC1) and its in vitro activities against $\mathrm{H}$. pylori motility and adherence. Biosci Biotechnol Biochem 2012;76:850-2.

12. Deguchi R, Nakaminami H, Rimbara E, Noguchi N, Sasatsu M, Suzuki T, et al. Effect of pretreatment with lactobacillus gasseri oll2716 on first-line Helicobacter pylori eradication therapy. J Gastroenterol Hepatol 2012;27:888-92.

13. Ojetti V, Bruno G, Ainora ME, Gigante G, Rizzo G, Roccarina D, et al Impact of Lactobacillus reuteri supplementation on anti-Helicobacter pylori levofloxacin-based second-line therapy. Gastroenterol Res Pract 2012;2012:740381.

14. Manfredi M, Bizzarri B, Sacchero RI, Maccari S, Calabrese L, Fabbian F, et al. Helicobacter pylori infection in clinical practice: Probiotics and a combination of probiotics + lactoferrin improve compliance, but not eradication, in sequential therapy. Helicobacter 2012;17:254-63.

15. Medeiros JA, Goncalves TM, Boyanova L, Pereira MI, de Carvalho JN, Pereira AM, et al. Evaluation of Helicobacter pylori eradication by triple therapy plus lactobacillus acidophilus compared to triple therapy alone. Eur J Clin Microbiol Infect Dis 2011;30:555-9.

16. Yoon H, Kim N, Kim JY, Park SY, Park JH, Jung HC, et al. Effects of multistrain probiotic-containing yogurt on second-line triple therapy for Helicobacter pylori infection. J Gastroenterol Hepatol 2011;26:44-8.

17. Zou J, Dong J, Yu X. Meta-analysis: Lactobacillus containing quadruple therapy versus standard triple first-line therapy for Helicobacter pylori eradication. Helicobacter 2009;14:97-107.

18. Wang ZH, Gao QY, Fang JY. Meta-analysis of the efficacy and safety of Lactobacillus-containing and Bifidobacterium-containing probiotic compound preparation in Helicobacter pylori eradication therapy. J Clin Gastroenterol 2013;47:25-32. 
19. Juni P, Witschi A, Bloch R, Egger M. The hazards of scoring the quality of clinical trials for meta-analysis. JAMA 1999;282:1054-60.

20. The Cochrane Collaboration. Cochrane Handbook for Systematic Reviews. Available at: http://www.cochrane.org/training/ cochrane-handbook/ (Version current at March, 2011).

21. Guyatt GH, Oxman AD, Schunemann HJ, Tugwell P, Knottnerus A. Grade guidelines: A new series of articles in the journal of clinical epidemiology. J Clin Epidemiol 2011;64:380-2.

22. Guyatt GH, Oxman AD, Vist GE, Kunz R, Falck-Ytter Y, Alonso-Coello $\mathrm{P}$, et al. Grade: An emerging consensus on rating quality of evidence and strength of recommendations. BMJ 2008;336:924-6.

23. Sykora J, Valeckova K, Amlerova J, Siala K, Dedek P, Watkins S, et al. Effects of a specially designed fermented milk product containing probiotic lactobacillus casei $\mathrm{dn}-114001$ and the eradication of $\mathrm{H}$. pylori in children: A prospective randomized double-blind study. J Clin Gastroenterol 2005;39:692-8

24. Goldman CG, Barrado DA, Balcarce N, Rua EC, Oshiro M, Calcagno ML, et al. Effect of a probiotic food as an adjuvant to triple therapy for eradication of Helicobacter pylori infection in children. Nutrition 2006;22:984-8.

25. Myllyluoma E, Veijola L, Ahlroos T, Tynkkynen S, Kankuri E, Vapaatalo $\mathrm{H}$, et al. Probiotic supplementation improves tolerance to Helicobacter pylori eradication therapy- a placebo-controlled, double-blind randomized pilot study. Aliment Pharmacol Ther 2005;21:1263-72.

26. Canducci F, Armuzzi A, Cremonini F, Cammarota G, Bartolozzi F, Pola P, et al. A lyophilized and inactivated culture of lactobacillus acidophilus increases Helicobacter pylori eradication rates. Aliment Pharmacol Ther 2000;14:1625-9.

27. Lionetti E, Miniello VL, Castellaneta SP, Magista AM, de Canio A, Maurogiovanni G, et al. Lactobacillus reuteri therapy to reduce side-effects during anti-Helicobacter pylori treatment in children: a randomized placebo controlled trial. Aliment Pharmacol Ther 2006;24:1461-8.

28. Lau J, Ioannidis JP, Terrin N, Schmid CH, Olkin I. The case of the misleading funnel plot. BMJ 2006;333:597-600.

29. Tong JL, Ran ZH, Shen J, Zhang CX, Xiao SD. Meta-analysis: The effect of supplementation with probiotics on eradication rates and adverse events during Helicobacter pylori eradication therapy. Aliment Pharmacol Ther 2007;25:155-68.

30. Chi CH, Lin CY, Sheu BS, Yang HB, Huang AH, Wu JJ. Quadruple therapy containing amoxicillin and tetracycline is an effective regimen to rescue failed triple therapy by overcoming the antimicrobial resistance of Helicobacter pylori. Aliment Pharmacol Ther 2003;18:347-53.
31. Kao AW, Cheng HC, Sheu BS, Lin CY, Sheu MJ, Yang HB, et al. Posttreatment 13C-urea breath test is predictive of antimicrobial resistance to H. pylori after failed therapy. J Gen Intern Med 2005;20:139-42.

32. Chey WD, Wong BC. American College of Gastroenterology guideline on the management of Helicobacter pylori infection. Am J Gastroenterol 2007; 102:1808-25.

33. Collado MC, Gonzalez A, Gonzalez R, Hernandez M, Ferrus MA, Sanz Y. Antimicrobial peptides are among the antagonistic metabolites produced by bifidobacterium against Helicobacter pylori. Int J Antimicrob Agents 2005;25:385-91

34. Aiba Y, Suzuki N, Kabir AM, Takagi A, Koga Y. Lactic acid-mediated suppression of Helicobacter pylori by the oral administration of Lactobacillus salivarius as a probiotic in a gnotobiotic murine model. Am J Gastroenterol 1998;93:2097-101.

35. Hsieh PS, Tsai YC, Chen YC, Teh SF, Ou CM, King VA. Eradication of Helicobacter pylori infection by the probiotic strains Lactobacillus johnsonii mh-68 and L. Salivarius ssp. Salicinius ap-32. Helicobacter 2012;17:466-77.

36. Michetti P, Dorta G, Wiesel PH, Brassart D, Verdu E, Herranz M, et al Effect of whey-based culture supernatant of Lactobacillus acidophilus (johnsonii) La1 on Helicobacter pylori infection in humans. Digestion 1999;60:203-9.

37. Smoot DT. How does Helicobacter pylori cause mucosal damage? Direct mechanisms. Gastroenterology 1997;113:S31-4,S50.

38. Kabir AM, Aiba Y, Takagi A, Kamiya S, Miwa T, Koga Y. Prevention of Helicobacter pylori infection by lactobacilli in a gnotobiotic murine model. Gut 1997;41:49-55.

39. Nam H, Ha M, Bae O, Lee Y. Effect of Weissella confusa strain pl9001 on the adherence and growth of Helicobacter pylori. Appl Environ Microbiol 2002;68:4642-5.

40. Byrd JC, Yunker CK, Xu QS, Sternberg LR, Bresalier RS. Inhibition of gastric mucin synthesis by Helicobacter pylori. Gastroenterology 2000;118:1072-9.

41. Mack DR, Ahrne S, Hyde L, Wei S, Hollingsworth MA. Extracellular MUC3 mucin secretion follows adherence of lactobacillus strains to intestinal epithelial cells in vitro. Gut 2003;52:827-33.

42. Hwang SW, Kim N, Kim JM, Huh CS, Ahn YT, Park SH, et al. Probiotic suppression of the H. pylori-induced responses by conjugated linoleic acids in a gastric epithelial cell line. Prostaglandins Leukot Essent Fatty Acids 2012;86:225-31.

43. Rousseaux C, Thuru X, Gelot A, Barnich N, Neut C, Dubuquoy L, et al. Lactobacillus acidophilus modulates intestinal pain and induces opioid and cannabinoid receptors. Nat Med 2007;13:35-7. 